\title{
Note sur la définition des (c Sols Indurés Roches Tendres ) (SIRT)
}

\section{Groupe \\ de travail commun \\ CFGI-CFMR-CFMS}

Animé par A. GUILLOUX

Terrasol

72, avenue Pasteur 93108

Montreuil Cedex

a.guilloux@terrasol.com et composé de R. COJEAN

(ENPC-ENSMP),

M. DORE (Arcadis),

D. FABRE (Cnam),

M. GHOYRECHI (Inéris),

J.-B. KAZMIERCZAK (Inéris)

V. MERRIEN-SOUKATCHOF

(Laego),

J.-P. PIGUET (Andra),

F. ROPERS (Géodynamique

\& Structures),

J.-F. SERRATRICE (CETE

Aix-en-Provence)

J.-P. TISOT (ENSG),

T. YOU (Geostock)
Les géotechniciens se trouvent souvent confrontés à des terrains dont le comportement est intermédiaire entre celui des sols et des roches, et qui sont de ce fait difficiles à traiter avec les approches classiques. Les trois Comités français de géologie de l'ingénieur, de mécanique des sols et de mécanique des roches ont donc mis en place un groupe de travail chargé de réfléchir à cette problématique transversale. Cette note technique présente le fruit de leurs premières réflexions sur la définition des SIRT, qui servira de base pour les futures réflexions sur leur analyse comportementale et sur les applications pratiques aux sites naturels ou ouvrages de génie civil.

Mots-clés : géologie de l'ingénieur, mécanique des sols, mécaniques de roches, sols indurés, roches tendres.

\section{Note on ( Hard Soils Soft Rocks ) (HSSR) definition}

Geotechnical engineers' have frequently to face to grounds with. an intermediate behaviour between soils and rocks. The three French cormmittees on Engineering Geology, Soil Mechanics and Rock Mechanics decided to launch a working group in charge of a reflection on this transversal problematic. This technical note presents its first thoughts on the HSSR definition, basis of the future work on the HSSR behaviour and their application to natural sites and civil construction.

Key words : engineering geology, soil mechanics, rock mechanics, hard soils, soft rocks. 


\section{Préambule}

Dans le cadre des actions communes aux trois Comités français de géologie de l'ingénieur, de mécanique des sols et de mécanique des roches, il est apparu que les terrains que l'on appelle couramment "sols indurés et roches tendres: SIRT » (en anglais : Hard Soils and Soft Rocks HSSR) constituaient un thème de réflexion tout à fait transversal.

En effet, ces terrains sont à la croisée des approches de ces trois disciplines, et il existe encore peu de littérature, notarnment en langue française, faisant une synthèse des problématiques spécifiques associées à ces terrains, que ce soit en termes de définition, de caractérisation ou d'application aux ouvrages de génie civil.

11 a donc été mis en place, en 2003, un groupe de travail chargé de réfléchir sur ce sujet, et dont l'ambition est à terme d'éditer un ouvrage. La première partie de la réflexion s'est orientée vers la définition même des SIRT, dont il est vite apparu qu'elle était loin d'être aussi évidente que le terme lui-même pouvait le laisser supposer.

Notre groupe de travail a ainsi rédigé un premier document portant sur la définition de ces SIRT, qu'il nous a paru utile de publier sous forme de note technique, afin de faire réagir la communauté géotechnique, de façon à pouvoir élargir la réflexion par les expériences de nombreux géotechniciens.

\section{2}

\section{Comment définir un SIRT?}

De par leur définition même les " sols indurés et roches tendres ) (SIRT) sont, bien sûr, à la frontière entre les sols et les roches, mais cette définition est loin d'être suffisante. Avant de travailler sur les aspects pratiques de caractérisation des SIRT et sur la façon dont ils doivent être abordés dans la conception, le dimensionnement et la construction des différents types d'ouvrages de génie civil, il convient de s'interroger sur leur définition même: qu'est-ce qu'on appelle un SIRT?

Différentes approches apparaissent a priori envisageables:

- une approche par la «résistance » puisque le terme même fait référence à cette notion:

- une approche géologique, en s'intéressant à la fois à la genèse, à la description et à la classification
- une approche (comportementale» en examinant les spécificités de comportement des SIRT, notamment en ce qui touche aux différences par rapport aux sols ou aux roches.

Nous balayons dans ce document ces différents types d'approches, pour tenter de dégager certains outils d'identification, de définition et si possible de classification des SIRT. L'objectif principal est d'aboutir à un langage commun, et d'identifier les principaux aspects du comportement communs à ces terrains.

\section{3}

\section{Principales différences de comportement entre les sols et les roches}

Avant de chercher à définir les SIRT, il apparait utile de s'interroger sur les principales différences de comportement entre les sols et les roches, afin de mettre en évidence les raisons pour lesquelles les SIRT ne peuvent en général être traités ni comme un sol, ni comme une roche.

Les sols comme les roches sont constitués, à Yéchelle de l'échantillon comme à l'échelle microscopique, d'un assemblage de grains dont la nature minéralogique est souvent comparable entre sols et roches. La différence entre ces deux types de matériaux est en fait essentiellement due aux forces « d'assemblage » entre ces grains: dans le cas des roches, ces forces sont de forte intensité et permanentes à l'échelle de temps humaine; dans le cas des sols, elles sont beaucoup plus faibles et peuvent varier dans le temps.

A l'échelle macroscopique, échelle à laquelle on s'intéresse habituellement pour les ouvrages, on peut différencier de façon schematique les comportements des sols et des roches comme suit :

- Un sol se comporte comme un milieu continu, qui peut être traité dans le cadre de la mécanique des sols. En particulier il est le plus souvent caractérisé par une loi de comportement de type élastoplastique, qui peut être correctement évaluée à partir d'essais sur des échantillons de petites dimensions (Fig. 1);

- Une roche au contraire est un milieu fortement discontinu, pour lequel les différents types de discontinuités structurales (plans de stratification, de fracturation, de foliation, etc.) jouent un rôle déterminant par rapport à celui de la matrice rocheuse elle-même. Ce schéma de blocs séparés par des discontinuités est la base de nombreuses approches en mécanique des roches (Fig. 2). 


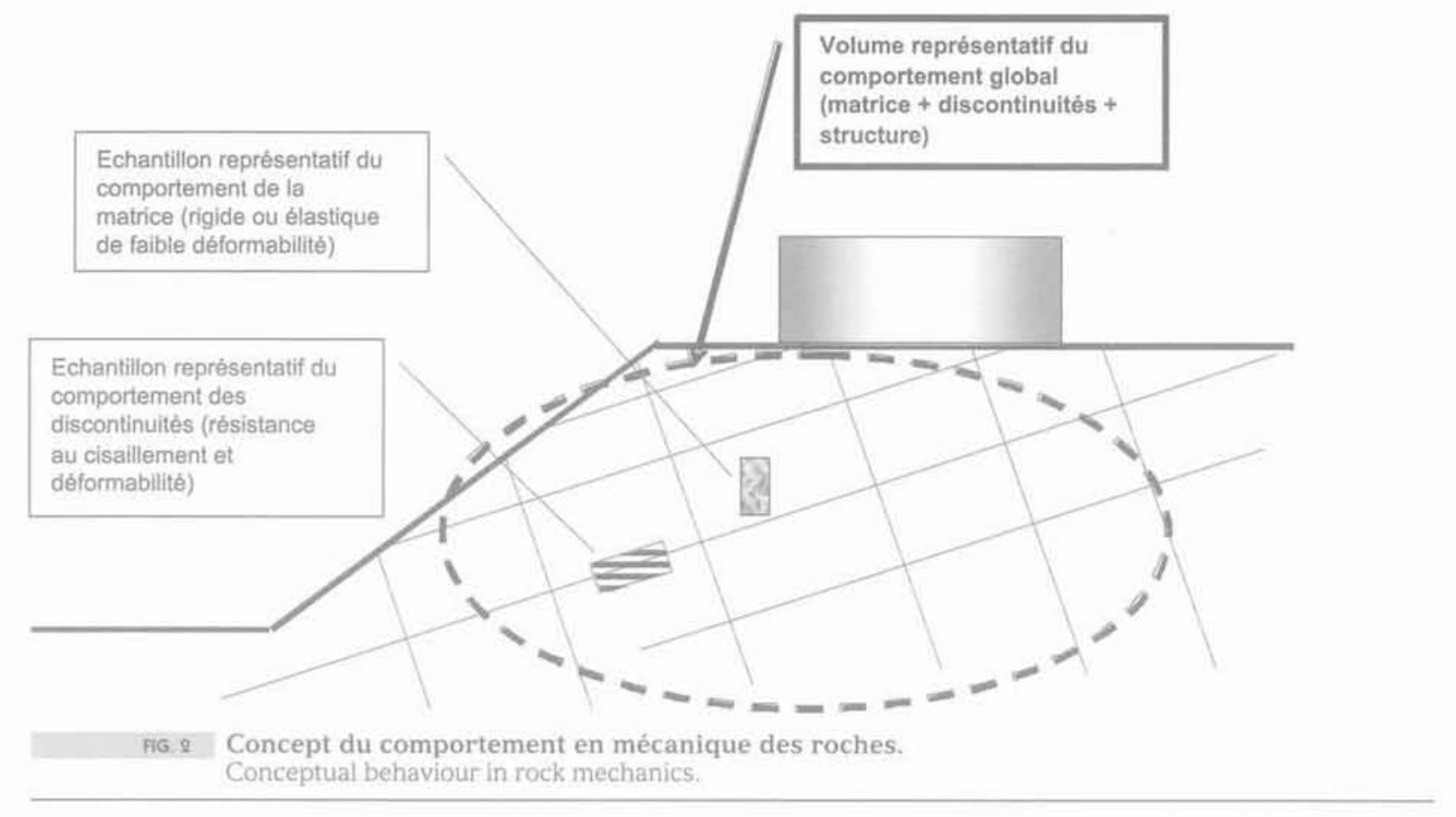

Leur caractérisation intègre donc à la fois :

- le comportement de la matrice, qui est analysé à partir d'essais de laboratoire sur des échantillons de petit volume;

- le comportement des discontinuités, également analysé à partir d'essais de cisaillement sur des échantillons de petites ou grandes dimensions, voire in situ, ainsi que par des essais hydrauliques;

- l'aspect structural, c'est-à-dire la répartition dans l'espace des différentes discontinuités, et ce à l'échelle du massif ou de l'ouvrage, c'est-à-dire d'ordre plurimétrique à pluridécamétrique (voire plus pour les grands ouvrages)

- enfin le comportement en grande masse, qui doit intégrer ces différents aspects, tant en termes de résistance que de déformabilité.

Dans de nombreux cas, et notamment pour les ouvrages peu profonds où les niveaux de contraintes sont modérés, ce comportement peut être simplifié par un modèle de blocs rigides (ou tout au moins restant dans le domaine élastique avec une déformabilité faible à négligeable) découpés par un réseau de discontinuités.

- Un SIRT se situe le plus souvent à l'intersection de ces deux types de comportement, c'est-à-dire dans un domaine où à la fois les influences de la structure et de la déformabilité de la matrice sont importantes. Ainsi pour les SIRT:

- le rôle de la structure (les discontinuités) y est souvent déterminant, et doit donc être pris en compte explicitement;

- et on ne peut se contenter d'un comportement de la matrice (les « blocs $»)$ parfaitement rigide ou même seulement élastique linéaire, mais il faut considérer qu'il peut atteindre le domaine de rupture et que sa déformabilité est non négligeable.

C'est pourquoi, même pour des ouvrages peu profonds avec des niveaux de contraintes modérés, le comportement d'un SIRT ne peut être traité ni par des approches purement «mécanique des sols », ni par des approches purement « mécanique des roches $\gg$.

\section{4}

\section{Approche géologique}

\section{1}

\section{Approche descriptive}

\section{Aftit}

\section{Description pétrographique à l'échelle de l'échantillon}

A partir des terrains que l'on peut, par expérience, certainement classer en SIRT, il est possible de mettre en évidence quelques aspects pétrographiques qui pourraient être des caractéristiques communes à certains SIRT.

\section{Alit}

Minéralogie

Certains terrains ont un comportement de SIRT parce qu'ils possèdent certains minéraux en proportion importante:

- des minéraux argileux, correspondant à des formations telles que: argiles raides, pélites, argilites, schistes, marnes et certains marno-calcaires, arènes granitiques, molasses argilo-marneuses...

- des minéraux de faible dureté ou forte déformabilité, correspondant à des formations telles que: gypse, sel, charbon...

Cette classification minéralogique apparaît pertinente pour certains SIRT, mais à l'évidence elle ne peut se suffire en elle-même, notamment parce qu'on ne peut éviter de faire appel à des notions complémentaires de structure des terrains. En outre certains SIRT ont un comportement spécifique, bien que leurs composants minéralogiques soient majoritairement de dureté moyenne à forte (calcite, quartz), du fait d'une structure microscopique particuliere; c'est notamment le cas particulier des craies évoquées plus loin. 


\section{1:4h:}

\section{Structure}

Bien qu'il ne soit pas possible de définir un terrain uniquement par sa structure, il faut souligner ici combien une très bonne description de type géologique est essentielle dans les cas des SIRT. En effet, une bonne caractérisation des SIRT, notamment en terme d'identification, apparait essentielle pour bien en appréhender le comportement.

Parmi les paramètres d'identification importants, il convient de citer:

- la granulométrie, qui constitue bien sûr un élément de base, et dont il convient de préciser l'échelle à laquelle se fait la détermination (en laboratoire sur échantillon par tamisage, ou au microscope pour les formations rocheuses);

- la texture, c'est-à-dire l'arrangement des grains:

- la porosité, pour laquelle il convient de distinguer:

- la porosité de pores, a priori inférieure à $25 \%$ dans les roches, et ne dépassant que très rarement $50 \%$ dans les sols;

- la porosité de fissures, beaucoup plus faible (le plus souvent inférieure à $1 \%$ ), mais qui peut jouer un rôle tout aussi déterminant;

- mais aussi la morphologie de cette porosité (répartition des tailles des pores, continuités entre pores...). Une telle approche physique peut permettre d'expliquer le comportement parfois atypique de certains SIRT.

Cette description en laboratoire, doit se faire à différentes échelles:

- à l'échelle macroscopique de l'échantillon: nature, taille et forme des grains, porosité, liaisons entre grains et leur nature (argileuse, calcaire, quartzitique, etc ...), microfracturation. On peut citer notamment le cas des argiles surconsolidées mais microfissurées, des calcaires et grès tendres, des tufs volcaniques, etc.;

- à l'échelle microscopique : nature et arrangement des minéraux et des liaisons entre eux, orientations...; on peut citer particulièrement le cas des craies.

L'ensemble de ces paramètres a en effet de fortes implications sur le rôle des fluides interstitiels dans le comportement des terrains, avec en premier lieu l'eau, mais aussi l'air pour les sols non saturés

C'est en effet par cette description, qui devra être à la fois qualitative et quantitative, que l'on pourra comprendre et appréhender certains traits caractéristiques de leur comportement et de leur évolution possible dans le temps notamment.

\section{Mat to}

\section{Analyse géologique et structurale à l'échelle du massif}

Dans le cas des SIRT, comme d'ailleurs dans les approches de mécanique des roches, la description à l'échelle de l'échantillon, le plus souvent suffisante pour les terrains homogènes, devient en général insuffisante, car elle ne permet pas d'intégrer les hétérogénéités à l'échelle métrique ou plurimétrique, qui conditionnent fréquemment le comportement des SIRT.

C'est ainsi que la description géologique à l'échelle de l'échantillon devra être complétée par une description géologique à l'échelle du massif, à la fois pour la lithologie et les discontinuités majeures: failles, fracturations, stratification et épaisseur des bancs, schistosité et foliation, granularité incluant les éventuels blocs de grandes dimensions. Parmi les SIRT où cette description est indispensable, on peut citer notamment le cas des flysch, mylonites, moraines, alternances marnocalcaires, argiles microfissurées, etc.

\section{Synthèse sur la description géologique}

L'ensemble des ces réflexions permet de mettre en évidence deux notions importantes pour la définition et la description des SIRT:

- Une approche combinée « minéralogie et porosité », qui peut caractériser l'échelle de l'échantillon: on peut présenter le domaine des SIRT, ainsi que celui des sols et des roches, dans un diagramme triangulaire noté NAQ (Fig. 3) où un terrain peut être représenté par un point en fonction des valeurs relatives de la porosité totale $n$, de la teneur volumique A en minéraux «sensibles $n$ (argiles, gypse, sel, charbon) et de la teneur volumique $\mathrm{Q}$ en autres minéraux (quartz, calcite...), avec:

$$
\mathrm{n}+\mathrm{A}+\mathrm{Q}=100 \%
$$

Dans un tel diagramme les SIRT occupent une place importante, avec une porosité en majorité inférieure à $25 \%$ comme dans les roches, mais une teneur importante en "minéraux sensibles ». La zone des craies s.S. échappe au zonage précédent car le minéral présent (la calcitel n'est pas en cause dans l'explication du comportement, mais bien sa microstructure; les craies peuvent en fait se caractériser comme une roche à très forte porosité.

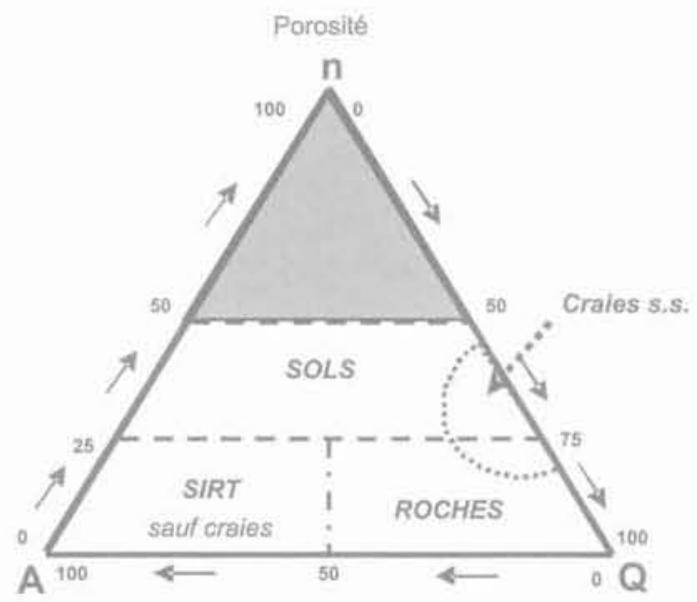

Minéraux sensibles Autres minéraux

FG. 3 Diagramme triangulaire «NAQ ». NAQ triangular diagram.

- La notion d'échelle du a volume élémentaire représentatif, VER n, qui est la dimension nécessaire pour pouvoir décrire le terrain et donc apprécier son comportement en intégrant tous les aspects structuraux: ainsi pour une craie homogène non fissurée, le VER pourra être d'ordre millimétrique voire moins, tandis que pour un flysch il sera d'ordre plurimétrique voire pluridécamétrique. 


\section{Genèse et types de formations géologiques}

On pourrait également tenter de définir une liste des terrains que l'on peut, par expérience, classer en SIRT. Une telle liste serait à l'évidence non exhaustive et, par ailleurs, il existe beaucoup d'appellations locales que l'on ne saurait faire entrer dans une telle liste sans risquer d'en oublier un grand nombre.

En revanche, une autre approche, également intéressante, consiste à s' intéresser à la genèse des terrains que l'on peut qualifier de SIRT. En effet, on peut mettre en évidence deux principaux modes de formation: - à partir de sols meubles, qui ont subi une induration progressive soit par compaction et/ou cimentation (diagenese $^{(i)}$ de boues argileuses ou calcaires: argilites ${ }^{(i)}$,

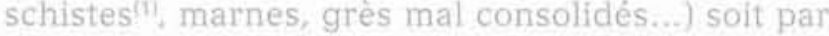
cimentation seule (sables de plage grésifiés, croûtes calcaires, tufs volcaniques...), mais sans avoir atteint une lithification ${ }^{(1)}$ complète (Fig. 4);

- à partir de roches dures, qui ont subi une altération progressive chimique et/ou mécanique, mais sans avoir atteint l'état de sol meuble (Fig. 5).

(1) Voir glossaire en fin d'article.
Cette approche ne permet pas, en revanche, d'intégrer facilement certains terrains d'origine diagénétique ou de «néo-formation $»$.

\section{5}

\section{Approche mécanique}

\section{1}

\section{Approche par la "résistance"}

En mécanique des roches le paramètre de base de nombreuses classifications est la résistance en compression uniaxiale $\sigma_{N}$, même s'il est communément admis que ce paramètre doit être utilisé avec précaution, car il n'est généralement pas représentatif du comportement global (effet des discontinuités) et que l'échantillonnage peut souvent perturber les analyses statistiques (on ne fait souvent les essais que sur les échantillons où l'on sait tailler une éprouvette, et donc a priori parmi ceux de meilleure qualité !).

En mécanique des sols, les paramètres caractéristiques de la résistance sont la cohésion et le frottement. Toutefois, les sols indurés présentent toujours une certaine « tenuè », c'est-à-dire que l'on peut en général en

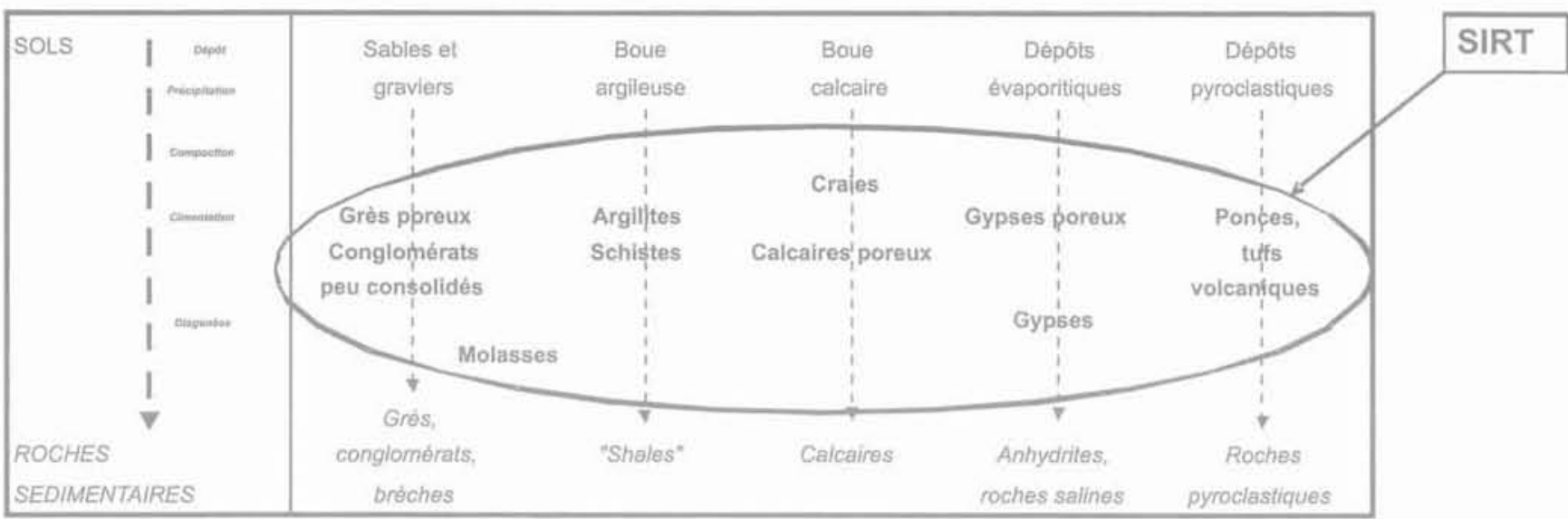

FIG 4 Genèse par induration.

Formation by a hardening $n$

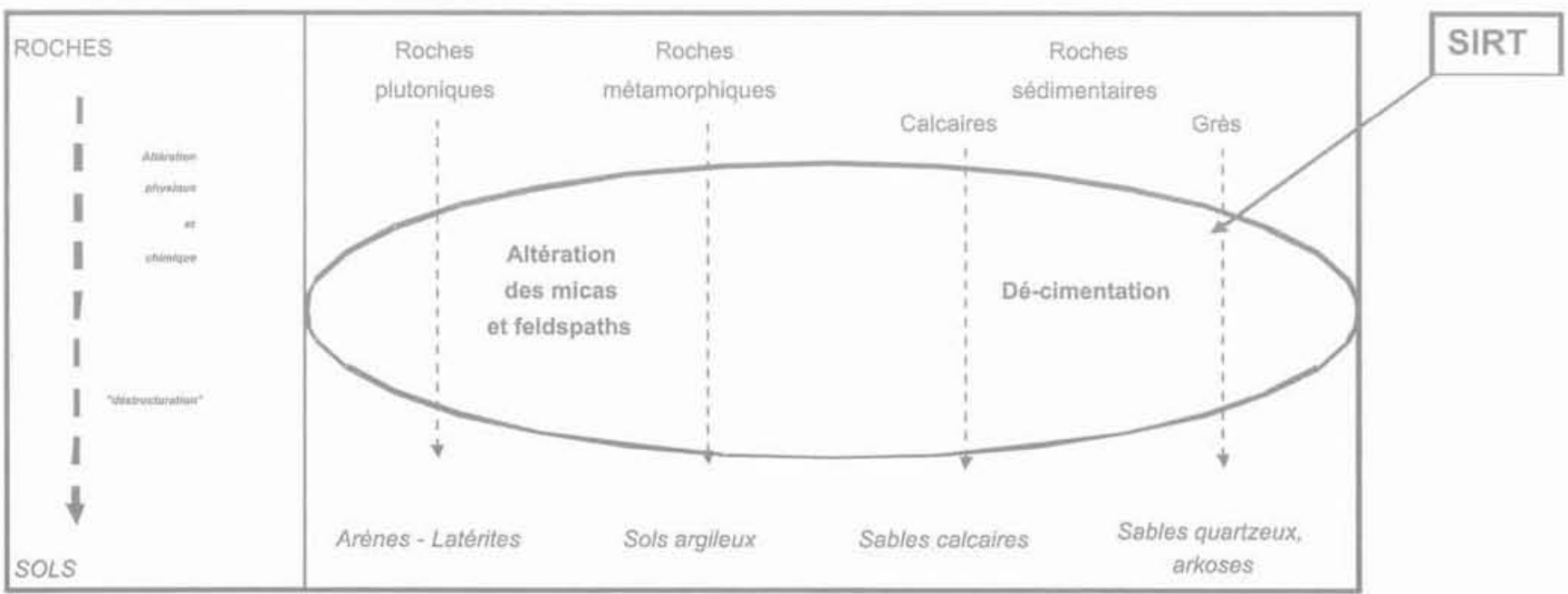


découper des éprouvettes, et donc faire des essais de résistance en compression uniaxiale.

Cette résistance en compression $\sigma$ peut donc sembler constituer un paramètre intéressant de définition des SIRT.

Si on s'intéresse alors aux classifications usuelles de mécanique des roches ou des sols, on peut chercher d'une part à se rattacher par exemple à la classification AFTES pour la première (Tableau I) et à celle de la norme NF EN ISO 14688-12 pour la seconde en l'adaptant de façon à transformer les classes de cohésion non drainée $c_{4}$ en classes de résistance en compression $\sigma$ par la relation $\sigma_{c}=2^{*} c_{u}$ (Tableau II).

On pourrait ainsi proposer de considérer comme SIRT potentiel:

- les roches de résistance faible à extrêmement faible: $\sigma_{c}<25 \mathrm{MPa}$ (classes AFTES RC5 à RC7);

- les sols de consistance très raicie à dure: $\sigma_{c}>0,3 \mathrm{MPa}$ et ainsi de définir globalement les SIRT comme les terrains de résistance en compression $\sigma_{c}$ comprise entre 0,3 et $25 \mathrm{MPa}$ approximativement.

\begin{tabular}{|c|c|c|}
\hline & \multicolumn{2}{|c|}{$\begin{array}{l}\text { Roches (classification Aftes 2003). } \\
\text { Rocks (Aftes } 2003 \text { classification). }\end{array}$} \\
\hline Classe & $\begin{array}{l}\text { Résistance } \sigma_{c} \\
(\mathrm{MPa})\end{array}$ & Description \\
\hline $\begin{array}{l}\text { RC1 } \\
\text { RC2 } \\
\text { RC3 } \\
\text { RC4 } \\
\text { RC5 } \\
\text { RC6 } \\
\text { RC7 }\end{array}$ & $\begin{array}{l}>200 \\
100 \text { à } 200 \\
50 \text { à } 100 \\
25 \text { à } 50 \\
5 \text { à } 25 \\
1 \text { à } 5 \\
<1\end{array}$ & $\begin{array}{l}\text { Résistance extrèmement élevée } \\
\text { Résistance très élevée } \\
\text { Résistance élevée } \\
\text { Résistance moyenne } \\
\text { Résistance faible } \\
\text { Rèsistance très faible } \\
\text { Résistance extrêmement faible }\end{array}$ \\
\hline
\end{tabular}

TABLEAU II Sols (adapté d'après la classification Afnor), Solls (from Afnor classification).

\begin{tabular}{lc} 
Classe & $\mathrm{o}_{\mathrm{e}}=2^{\prime} \mathrm{cu}(\mathrm{MPa})$ \\
\hline Dur & $>0,6$ \\
Très raice & 0,3 à 0,6 \\
Raicle & 0,15 à 0,3 \\
Ferme & 0,08 à 0,15 \\
Mou & 0,04 à 0,08 \\
Très mou & $<0,04$
\end{tabular}

Cette approche est loin d'être suffisante, notamment parce qu'elle ne caractérise que la résistance d'un milieu "homogène, isotrope et continu» sans considérer:

- les effets de la fragilité des terrains, qui peuvent être abordés par exemple par la notion de résistance en traction:

- les aspects de déformabilité, qui peut ètre abordée par le module de déformation: les roches de classe DE5 à DE6 au sens de l'Aftes peuvent souvent être considérées comme des SIRT;

- les effets de l'anisotropie;

- la présence de discontinuités, qui jouent pourtant un rôle fondamental.

\footnotetext{
(2) Reconnaissance et essais géotechniques. Dénomination, description
} et classification des sols, Partie 1: «Dénomination et description 1.

\section{Approche comportementale}

On rappelle que, pour la plupart, les SIRT se situent à la frontière entre deux types de comportement (de type sols, milieu continu, ou roche, milieu discontinu), avec un rôle souvent important joué par les discontinuités, mais également une matrice qui ne peut pas être considérée comme de «résistance élevée».

Il serait possible de résumer la problématique, en disant que les SIRT sont des terrains:

- trop hétérogènes pour ètre traités par les approches de la mécanique des sols;

- de résistance trop faible ou de déformabilité trop forte pour être traités par les approches de la mécanique des roches.

En outre, un grand nombre de SIRT présentent différents aspects de comportements particuliers, dont l'influence est déterminante sur les mécanismes mis en jeu dans les ouvrages, et qu'il faut donc prendre en compte par des caractérisations spécifiques. Sans chercher à en dresser une liste exhaustive, on peut citer les aspects suivants:

- L'hétérogénéité et l'anisotropie souvent très prononcées (alternance de bancs «durs») et «tendres»), blocs emballés dans une matrice...). On retrouve ici la notion très importante de volume élémentaire représentatif, déjà évoquée au \$ 4.1.3, c'est-à-dire du volume de ("l'échantillon a décrire et analyser pour pouvoir caractériser un comportement du terrain représentatif du milieu agissant sur l'ouvrage et du problème mécanique étudié.

Par exemple, pour un talus de $20 \mathrm{~m}$ de hauteur dans une craie peu fracturée, le volume élémentaire représentatif pourra être décimétrique, alors que pour le même talus dans un flysch, il sera au moins plurimétrique.

On peut souligner à ce sujet que la vitesse de propagation des ondes sismiques mesurée in situ (en surface ou en forages) est un paramètre particulièrement intéressant, car il intègre à la fois la qualité de la matrice et l'effet des discontinuités;

- Des comportements intrinsèques aux terrains et liés à une certaine évolutivitér(3) ou sensibilité(2) à différents agents (variations de teneur en eau, de température), qui peuvent conduire à une altération physicochimique, au sens large, des terrains. Ces mécanismes, souvent liés à la minéralogie, se traduisent sur le plan du comportement soit par une réduction des caractéristiques mécaniques (perte de cohésion sous l'effet du gel), soit par des variations de volume (exemple du gonflement de certains minéraux argileux). Différents types d'essais permettent de caractériser cette plus ou moins grande sensibilité: essais d'altérabilité, de gélivité, de gonflement, voire de fragmentabilité pour les matériaux utilisés en remblais;

- Des comportements liés aux types de sollicitations appliquées, qui existent dans les tous les terrains, mais dont le rôle est souvent nettement plus déterminant pour les SIRT. On citera notamment:

- le confinement ou niveau de contraintes, qui influe directement sur les propriétés de résistance (courbe intrinsèque non linéaire) et de déformabilité (élasticité

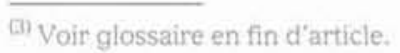


non linéaire, rôle des joints qui agissent plus ou moins selon le niveau de contraintes);

- les pressions interstitielles et, d'une façon plus générale, l'action de l'eau et autres fluides présents (air notamment);

- l'influence du temps à l'échelle humaine, avec les mécanismes de fluage (par exemple dans les marnes, argilites, gypses, etc.), qui se traduisent par des réductions significatives de résistance ou de déformabilité, ou les effets transitoires de pression interstitielle. Les mécanismes de gonflement ou d'altération rapide sont également caractéristiques de certains SIRT: on peut citer l'exemple des schistes cartons, qui peuvent très rapidement passer de la classe Aftes RC 4 à la classe RC 5, voire évoluer en « sols».

Pour ces différentes raisons, les classifications de roches (RMR, Q, GSI...) s'avèrent le plus souvent non applicables aux SIRT, notamment parce que le poids de la résistance de la matrice y est très faible (de l'orcire de $15 \%$ au maximum de la note globale pour le RMR), mais aussi parce que certains comportements particuliers n'y sont en général pas intégrés.

La question est alors de savoir si on peut envisager une classification dédiée aux SIRT, entièrement nouvelle ou basée sur des cadres existants (GSI par exemple), permettant à la fois de qualifier les terrains, mais aussi d'en déduire des caractéristiques géomécaniques moyennes (c, $\phi, E .$.$) représentatives du milieu global, au prix d'une$ homogénéisation (question d'autant plus cruciale quand ces milieux sont fortement hétérogènes)?

Ce sujet doit être au cceur de la réflexion sur la caractérisation des SIRT, qui fera l'objet de la suite des réflexions du groupe de travail.

\section{6}

\section{Synthèse: approche multicritère}

\section{1}

\section{Analyse multicritère}

Il apparait finalement qu'aucune des approches précédentes, prise indépendamment des autres, ne permet de définir un SIRT de façon absolue. Si la résistance, caractérisée par exemple par la résistance en compression $\sigma_{e}$ peut être un premier élément pour suspecter des SIRT, ce sont bien plutôt des types particuliers de comportement qui sont à I'origine de la spécificité des SIRT.

On peut toutefois retenir que le critère purement mécanique basé sur la résistance en compression uniaxiale $\sigma_{c}$ est pertinent, au moins pour les approches de type mécanique des sols, car il signifie que les méthodes usuelles d'essais de mécanique des sols ne sont plus applicables. Il faut toutefois garder à l'esprit que la valeur de $\sigma_{c}$ doit être analysée sur une population significative, de façon à en apprécier la variabilité, et qu'elle peut être largement dépendante de la teneur en eau, de la direction de l'échantillon par rapport à la structure, et également de la vitesse d'essai.

De même, pour les approches de type mécanique des roches, un tel critère basé sur la valeur de $\sigma_{c}$ permet d'alerter le géotechnicien sur le fait que les essais courants de mécanique des roches ne sont pas toujours suffisants pour appréhender certains types de comportements qui sont essentiels pour les SIRT (rôle des pressions interstitielles par exemple).

On pourra donc retenir le critère o comme un «signal d'alerte permettant d'attirer l'attention sur le fait que les procédures classiques d'essais de mécanique des sols ou des roches ne sont pas applicables pour les SIRT.

La structure et la lithologie des terrains sont d'autres éléments importants, qui peuvent conduire à définir différentes catégories de SIRT présentant chacun des analogies de comportement:

- les terrains à forte porosité;

- les terrains hétérogènes dans lesquels l'échelle du volume élémentaire représentatif est au moins métrique; - les terrains dont le constituant minéral majoritaire possède un comportement très particulier: les roches argileuses, les roches salines (gypse, sel gemme), le charbon (qui passe d'une roche à un sol);

- les terrains évolutifs à l'échelle de temps humaine.

Ce n'est en fait qu'une analyse multicritères qui peut permettre d'identifier un SIRT. Différents critères apparaissent ainsi réellement discriminants, et l'on peut proposer des fourchettes de valeurs de ces paramètres correspondant aux SIRT (Tableau III).

Dans cette approche multicritères, il est proposé que soit classé comme SIRT tout terrain vérifiant au moins deux de ces critères.

\section{2}

\section{Démarche d'identification des SIRT}

Nous proposons donc la démarche suivante pour l'identification et la caractérisation d'un SIRT lors des études de projets:

- On considérera qu'un terrain caractérisé par une résistance en compression o comprise entre 0,3 $\mathrm{MPa}$ et $25 \mathrm{MPa}$ peut être a priori considéré comme un SIRT: classes Aftes "roches RC5 à RC7 » ou classes Afnor " sols très raides à durs 》).

- Il est alors indispensable de compléter cette classification par d'autres approches, dès les phases préliminaires des études, pour qualifier plus précisèment cette notion de SIRT:

TABLEAU III Critères de définition d'un SIRT (2 critères au moins doivent être vérifiés).

Definition criteria for HSSR (2 criteria at least have to be chekched).

\begin{tabular}{|c|c|c|c|c|c|c|}
\hline Critère & $\begin{array}{c}\text { Résistance } \\
\sigma_{f} \text { (MPa) }\end{array}$ & $\begin{array}{c}\text { Porosité de } \\
\text { pores } n_{p}\end{array}$ & $\begin{array}{c}\text { Vitesse } \\
\text { sismique } \\
\text { in } s i t u V_{p}(m / s)\end{array}$ & Minéralogie & $\begin{array}{l}\text { Échelle du } \\
\text { volume élémentaire } \\
\text { représentatif }\end{array}$ & Évolutivité \\
\hline $\begin{array}{l}\text { Valeurs caractéristiques } \\
\text { pour un SIRT }\end{array}$ & 0,3 à $25 \mathrm{MPa}$ & $>25 \%$ & $\begin{array}{c}1200 \mathrm{à} \\
2500 \mathrm{~m} / \mathrm{s}\end{array}$ & $\begin{array}{l}\text { Plus de } 60 \% \text { de sel, } \\
\text { gypse, ou charbons } \\
\text { Plus de } 40 \% \text { de } \\
\text { minéraux argileux }\end{array}$ & $\begin{array}{l}\text { Métrique à } \\
\text { plurimétrique }\end{array}$ & $\begin{array}{l}\text { A l'échelle } \\
\text { humaine }\end{array}$ \\
\hline
\end{tabular}


- caractérisation lithologique et minéralogique;

- identification de la genèse :

- description géologique détaillée, qualitative et quantitative: discontinuités, hétérogénéité (alternances de bancs de nature et dureté contrastées, blocs emballés dans une matrice). Cette description doit se faire à l'échelle du massif global (pluridécamétrique) et de l'échantillon (décimétrique), voire dans certains cas à l'échelle microscopique:

- caractérisation par les paramètres d'identification (granularité, porosité)

Ces descriptions doivent permettre de préciser si deux, au moins, des critères du tableau III sont vérifiés, ce qui confirmera sa classification comme SIRT.

- En fonction de ces descriptions complémentaires, il sera alors nécessaire de bien identifier les particularités de comportement qui sont à prendre en compte pour l'application aux ouvrages (cf. §. 5.2).

- On pourra alors définir des méthodes et techniques de reconnaissance et essais qui soient adaptées à ces particularités.

\section{3}

\section{Poursuite du travail}

A partir de ces éléments, la suite du travail du groupe consistera:

- d'une part à établir une base de données permettant d'affiner l'analyse multicritères, afin de vérifier ou modifier les valeurs représentatives pour les différents critères proposés;

- d'autre part à identifier et préciser les types et procédures d'essais adaptés aux spécificités des SIRT, et qui pourraient s'écarter notablement des normes usuelles pour les sols ou les roches.

\section{$\overline{7}$}

\section{Glossaire} (d’après Foucault et Raoult)

Argilite : roche argileuse sans litage net, peu stratifiée et/ou indurée par compaction.

Diagenèse : ensemble des processus qui affectent un dépôt sédimentaire et le transforment progressivement en roche sédimentaire solide; elle peut être biochimique ou physico-chimique.

Évaporite : dépôt riche en chlorures et sulfates alcalins, qui se forme par concentration et précipitation suite à une évaporation intense.

Flysch: formation sédimentaire détritique composée essentiellement d'un empilement de turbidites (dépôts hétérogènes composés de matériaux grossiers à argileux) ultérieurement tectonisée. Ils se présentent sous forme d'alternances de bancs gréseux à schisteux.

Lithification: transformation d'un sédiment meuble en roche sédimentaire consolidée par compaction et cimentation.

Molasse: formation sédimentaire détritique composée essentiellement d'un empilement de turbidites (cf. ci-dessus) et de couches terrigènes non turbiditiques (grès, conglomérats). Ils se présentent sous forme de niveaux comportant en proportions variables des grès, marnes et argiles.

Mylonite: au sens large toute roche plus ou moins finement broyée; résultant souvent de failles tectoniques.

Pélite: roche sédimentaire détritique à grains très fins.

Ponce: roche volcanique très poreuse.

Pyroclastique: s'applique aux accumulations de débris de roches magmatiques éjectées par les volcans.

Schiste: au sens strict désigne une roche métamorphique se débitant facilement en plaquettes, Par extension le terme recouvre toute roche se débitant en plaquettes, c'est-à-dire toute roche litée à grains très fins, argileuse ou marneuse, même d'origine sédimentaire.

Shale: mot anglais, largement utilisé en français, désignant toute roche schisteuse plus ou moins argileuse. On pourrait dire qu'il correspond à un schiste (au sens large) sédimentaire.

Tuf volcanique: roche volcanique formée par accumulation des projections volcaniques en fragments millimétriques, pouvant contenir des blocs ou des cendres, et plus ou moins cimentée sous l'action de l'eau.

Turbidite: couche de sédiments détritiques déposée en une seule fois par un courant de turbidité, le plus souvent quartzeux et parfois calcaires, et d’épaisseur dépassant rarement le mètre.

\section{Bibliographie}

Afnor-Recueil de normes des essais gèotechniques.

Aftes - Recommandations relatives à la caracterisation des massifs rocheux utiles à l'étude et à la réalisation des ouvrages souterrains. Tunnels et Ouvrages souterrains $n^{\circ} 177$, mai-juin 2003.

CFMR - Manuel de mécanique des roches Tome 1, Fondements. Presses de l'École des mines, 2000

Foucault A., Raoult J,-F. - Dictionnaire de géologie. Dunod, 2001, $5^{\text {e éd. }}$

Hoek E.. Bray J.W. - Rock slope engineering. Ind. Min. Metall., London, 1981. 3rd edition revised.

Hoek E., Brown E. - Underground excavation in rock. Ind. Min. Metall. London. 1980.

Hoek E., Brown E. - Practical estimation of rock mass strength. Int. Journal of
Rock Mech. And Min. Sc., vol. 34. 1997.

Hoek E., Kaiser P.K., Bawden W.F. - Support of underground excavation in hand rocks. Balkema, 1995

LAEG - Final report of Working Group on Soft Rocks and Endurated Soils in South America. September 2002

Kavvadas M.J. Anagnostopoulos A.G. - A framework for the mechanical behaviour of structured soils. Proc. Int. Symp. On Hard soils-Saft Rocks, Naples, vol. 2 1998

Krauter E. - General co-report session 1 geological and geotechnical features, investigations and classification. Proc. Int. Symp. on Geotechnical Engineering of Hard solls-Soft Rocks, Athens. Balkema, vol. 3, 1997 .

Marinos P.G. - General report session 1: hard soils-soft rocks: geological features with special emphasis to soit rocks Proc. Int. Symp. on Geotechnical Engineering of Hard soils-Soft Rocks; Athens Baikema, vol, 3, 1997.

Marinos P. Hoek E. - GSI : a geological friendly toll for rock mass strength estimation. Proc, GeoEng2000 Conference, Melbourne, 2000.

Serratrice J,-F. - Outils et procédures de caractérisation des sols indurés et des roches tendres: l'expérience du LRPC d'Aix-en-Provence. Colloque PARAM 2000 * Paramètres de calcul gèotech nique $n$ Ss la dir. de J.-P. Magnan. Presses de I'ENPC/LCPC, 2002.

Vaughan P.R. - Engineering behaviour of weak rocks : some answers and some question. Proc. Int. Symp, on Geotechnical Engineering of Hard soils-Soft Rocks, Athens, Balkema, vol. 3, 1997 